\title{
Deslocalización de la producción y la fuerza de trabajo: Bolivia - Argentina y las tendencias mundiales en la confección de indumentaria*
}

\author{
Delocalization of production and labour: Bolivia - Argentina and the \\ worldwide trends in garment industry
}

Paula Dinorah Salgado**

Universidad Nacional de Tres de Febrero, Buenos Aires, Argentina

Recibido: 21 de mayo de 2014. Aprobado: 9 de febrero de 2015

\begin{abstract}
Resumen
La industria de la confección de indumentaria ha reportado un notable crecimiento en la última década, acompañado de un aumento del trabajo altamente precarizado y carente de registro -laboral e impositivo-. Esta tendencia es resultado del desligamiento progresivo de la producción hacia talleres no registrados. Diversos factores explican esta coyuntura. El presente artículo analiza las transformaciones en la industria de la confección local en sintonía con los cambios que han operado a nivel mundial con el propósito de situar el caso argentino en el marco de las tendencias globales que ha seguido la industria de la indumentaria en las últimas décadas. Para ello se indaga la relación entre el desenvolvimiento del proceso productivo -fundamentalmente a partir de la desintegración vertical- y el incremento del empleo de migrantes -en su mayoría procedentes de Bolivia- indocumentados, con el crecimiento de las condiciones de trabajo extremadamente precarizadas.
\end{abstract}

Palabras claves: deslocalización, superexplotación, migración.

Una versión preliminar de este trabajo fue presentada en $3^{\circ}$ Jornadas Internacionales de Problemas Latinoamericanos organizadas por la Universidad Nacional de Cuyo (Mendoza, Argentina).

** Licenciada en Sociología (UBA), Maestranda en Metodología de la Investigación (UniBo-UNTreF). Doctoranda en Ciencias Sociales (UBA). Correo electrónico: pauladsalgado@yahoo.com.ar 


\begin{abstract}
Garment industry shows a prominent growth in the last decade, linked to an increase of highly precarious -and unregistered- work. This trend is the result of gradual detaching of labor-intensive activities to unregistered sewing shops. Various factors explain this situation. In this paper we analyze the transformations in local garment industry in line with the changes carried out worldwide, pursuing the goal of positioning Argentine case in the context of global trends followed by clothing industry in the last decades. For this purpose, we look into the relationship between the developments of the production process -related to the breakdown in the vertically-integrated mode of production-, the spread of the employment of migrants -in their majority from Bolivia- undocumented, and the rise of extremely precarious work conditions.
\end{abstract}

Keywords: delocalization, superexploitation, migration.

\title{
Introducción
}

En la actualidad, la industria de la confección ha trascendido como uno de los ámbitos productivos en los que se extiende una forma particular de explotación del trabajo denominada reducción a la servidumbre. Así lo revelan centenares de denuncias a talleres clandestinos y a marcas que los emplean. Otro ejemplo de su visibilidad es la extensión del término trabajo esclavo -tanto en el ámbito periodístico como en el académico- para caracterizar a la forma que asume este tipo de trabajo, fundamentalmente a partir del incendio en el taller de la calle Luis Viale en la ciudad de Buenos Aires en 2006 en el que murieron dos costureros y cuatro niños que vivían allí debido a que estaban encerrados y no pudieron escapar.

Este tipo de trabajo se extiende en sectores de gran vulnerabilidad: mayormente migrantes no registrados/as, muchos de los cuales han sido traídos al país mediante redes de trata. Producto de la irregularidad en que se ejerce este tipo de empleo, sumado a la carencia de registros migratorios, no existen datos oficiales a través de los que se pueda estimar la magnitud y el expansión de esta forma de explotación del trabajo en el ámbito de la confección de indumentaria. 
Para el presente análisis ${ }^{1}$ se ha realizado una triangulación de metodologías cuantitativa y cualitativa-, y de fuentes ${ }^{2}$. El objetivo ha sido tanto controlar las diversas estimaciones, como multiplicar las perspectivas que se arrojan sobre el fenómeno en estudio y que, en conjunto, permiten dar cuenta de gran cantidad de dimensiones de abordaje.

A continuación se expone en primera instancia una reseña de la teoría sobre la que se enmarca el análisis posterior. Seguidamente se desarrollan en diferentes apartados la historización de la industria de la indumentaria, las particularidades de la división del trabajo al interior de la rama, el desenvolvimiento de la migración Bolivia-Argentina para analizar, finalmente, cómo la convergencia de estos procesos da lugar a la superexplotación del trabajo como forma extendida en la industria de la confección de indumentaria.

\section{Consideraciones teóricas}

La industria de la indumentaria resulta un caso paradigmático para analizar la complejidad que reviste la relación capital-trabajo en la actualidad. En ella se aglutina un extenso gradiente de formas de explotación del trabajo que van desde los altos directivos de la industria de la moda al oficial de costura carente de registro laboral y migratorio. Producto de esta multiplicidad esta rama resulta una suerte de caso crítico en tanto recorre de extremo a extremo al amplio espectro de configuraciones del trabajo y el empleo presentes en el capitalismo actual. Decidimos centrar el análisis en las que cobran protagonismo a partir de la reestructuración capitalista de mediados de los años 70, particularmente, en el empleo de migrantes no registrados/as, muchos de los cuales se encuentran en condiciones de reducción a la servidumbre. Si bien, a nivel local, su expansión se inicia en la década de los 90, las condiciones que la han garantizado son resultado de las transformaciones estructurales operadas desde entonces. Esta mutación ha sido descrita por Harvey (2007) como el tránsito del fordismo a la acumulación flexible, caracterizado

1 Agradezco los comentarios y sugerencias del Dr. Pablo Ghigliani y del Dr. Agustín Santella que sirvieron para articular las reflexiones de este trabajo.

2 Se analizan estadísticas de población (Instituto Nacional de Estadística y Censos [INDEC], Dirección General de Estadísticas y Censos del Gobierno de la Ciudad de Buenos Aires), de trabajo (Ministerio de Trabajo y Seguridad Social [MTySS]), de industria (INDEC y MTySS), estimaciones del desenvolvimiento del trabajo no registrado (Instituto Nacional de Tecnología Industrial), así como datos procedentes de otros estudios académicos y gubernamentales. Las fuentes cualitativas consisten en entrevistas en profundidad realizadas a trabajadoras y trabajadores de la confección, empresarios de la rama, funcionarios estatales e informantes clave, así como denuncias realizadas a la Defensoría del Pueblo de la Ciudad Autónoma de Buenos Aires. 
por el pasaje de la producción en masa en cadena de montaje, la organización política de masas y el Estado interventor a la dispersión espacial de la producción vinculada a su descentralización, la reducción del Estado -ligada a la privatización y liberalización- y la búsqueda de mercados especializados.

Desde entonces el espacio se ha vuelto una dimensión donde el capital ha intervenido activamente a fin de satisfacer las necesidades de reducción de la masa destinada a la fuerza de trabajo -capital variable- y a los medios de producción -capital constante-. En otros términos, se trata del proceso de deslocalización industrial: desplazamiento de uno a otro territorio, en gran parte de los casos atravesando fronteras administrativas que suponen un régimen legal diferente -impositivo y/o laboral-, así como diferentes valuaciones monetarias. Todos estos factores han facilitado la reducción de la porción del capital destinada al pago del trabajo.

Son diversas las acepciones con que se ha descrito esta transformación desde la economía, la sociología y la geografía. Ello se debe a la multiplicidad de procesos que son enlazados, que se involucran unos a otros dando lugar a una metamorfosis en la arquitectura de la producción. Entre los que cobraron mayor trascendencia Hijzen, Görg y Hine (2003), destacan los siguientes: fragmentación, acuñado por Jones y Kierzkowski en 1990; especialización vertical desarrollado por Hummels, Ishii y Yi en 2001; especialización intra-producto propuesto por Arndt (1997) promediando los años 90; deslocalización utilizado por Leamer (1998) finalizando el siglo XX; y tercerización y desintegración ampliamente trabajados por Feenstra (1998) desde aquellos años. Fragmentación y desintegración refieren al estado anterior de la organización industrial característica del fordismo, una industria que integraba verticalmente sus diferentes etapas de producción. La especialización hace alusión a un pasado holístico, pero sin la connotación negativa de los otros conceptos. Por último, tercerización y deslocalización refieren a las derivaciones que implica la nueva forma de organización de la producción, a la vez que vuelve inteligible el lazo solidario entre las diferentes instancias en las que se divide el proceso productivo, vinculando las fases que concentran el trabajo intelectual -que se apropian de gran parte del plusvalor- con las actividades trabajo-intensivas -que suelen derivarse a regiones que permiten bajar el precio del salario-.

Baldone, Sdogati y Tajoli (2001) sostienen que la industria textil y de confección resulta un excelente ejemplo de integración económica entre países a través de la deslocalización internacional de la producción debido a que ha sido afectada por este proceso más que muchas otras ramas. A partir de datos de comercio exterior entre Europa Occidental, y Europa Central y Oriental, los autores señalan que se han llevado a cabo una 
serie de cambios en la organización de la producción, que dieron por resultado el traslado de las actividades trabajo-intensivas a países caracterizados por costos del trabajo relativamente menores, mientras se mantenían las fases fundamentales de creación y distribución de mercancías en los países a los que pertenecían las industrias.

Los autores se preguntan por los motivos que llevan a la elección de unos países por encima de otros para tercerizar ciertas fases del proceso productivo -mayormente las que concentran actividades trabajo-intensivas- y concluyen que los costos laborales, junto a la proximidad geográfica y cultural, son las razones más importantes para la elección primigenia de un país determinado con el objetivo de derivar parte de la producción ${ }^{3}$.

A la luz del caso argentino se identifica lo que hemos definido como un proceso de deslocalización en un triple sentido:

1. En términos de Estados-nación: el trabajo intelectual, el manual y la comercialización tienden a separarse y concentrarse en diferentes puntos del globo.

2. De las etapas del proceso productivo: su fragmentación ha implicado la centralización del trabajo intelectual y la dispersión trabajo manual, disgregándolo en una multiplicidad de pequeños talleres atomizados.

3. De la fuerza de trabajo: en búsqueda de mejores condiciones laborales trabajadores migran a nuevos destinos -en muchos casos de forma no regularizada-, exponiéndose a una situación de extrema vulnerabilidad.

A continuación proponemos un recorrido en línea con los ejes propuestos. Si bien esta propuesta teórica se manifiesta empíricamente como una múltiple convergencia de las dimensiones expuestas, fundamentamos nuestra decisión en la posibilidad que ofrece su abordaje individualizado en cuanto a la profundización y de visibilización de matices que aportan a la singularidad del fenómeno en estudio en el contexto seleccionado.

3 Se trata puntualmente del caso de Alemania analizado en 2001. En el mismo estudio se señala que otros países han tendido a elegir el país al que tercerizar los segmentos de mayor intensidad laboral dando prioridad al costo de la fuerza de trabajo. Este sería el caso de Italia. En la investigación se explica por el inicio tardío de la desintegración vertical en éste último país. 


\section{Separación del proceso de producción a escala planetaria}

El efecto más notorio de la desverticalización industrial es la separación de fases de la producción en diferentes Estados-nación. Esta desintegración es llevada a cabo junto a una integración comercial a escala planetaria: se trata de dos caras de un nuevo movimiento del capital que a medida que se expande y profundiza, incentiva canales de comunicación y circulación. En procura de la reducción de costos, se fragmenta el proceso productivo y diversas etapas son tercerizadas. Mayoritariamente se trata de actividades trabajo-intensivas y/o de aquéllas que utilizan gran cantidad de trabajo no calificado. Según Feenstra (1998), el crecimiento de la tercerización a nivel mundial durante la década de los 80 estuvo relacionado en parte con el desarrollo de la tecnología en las comunicaciones y con la velocidad en que la calidad y el diseño de los productos comenzaron a ser monitoreados. Silver (2005) destaca la importancia de establecer una distinción con la falta de integración vertical del pasado, en tanto en el presente "suele ser sólo una apariencia superficial" bajo la cual, el conjunto de pequeñas firmas responde a grandes corporaciones "que han subcontratado esas tareas para recortar costes y protegerse a sí mismos de la responsabilidad" (p. 193).

La Organización Internacional del Trabajo OIT advierte sobre las transformaciones acaecidas fundamentalmente en materia de empleo a partir de la década del 70, cuando comienza a operarse una traslación desde Europa y Estados Unidos hacia Asia y otros países de industrialización tardía. Esta tendencia está vinculada al desplazamiento desde el ámbito formal al informal, lo que ha repercutido en términos negativos en las remuneraciones, así como en las condiciones de trabajo:

muchos puestos de trabajo a tiempo completo en la fábrica se convirtieron en empleos temporales y a tiempo parcial, especialmente en las industrias del vestido y del calzado. Además aumentó el recurso al trabajo a domicilio y el que se efectúa en los pequeños talleres $(1996$, p. 3).

Promediando la década del 90 más del 60\% de las exportaciones de prendas de vestir eran fabricadas en países de industrialización tardía, siendo Asia el principal proveedor mundial -productor del 32\% en ese entonces-. En 1998, por primera vez, Asia fue responsable de más del 50\% de la producción mundial de vestimenta. Entre 1995 y 1998 la participación de las Américas en la producción mundial creció en un 14,3\%, incremento que se debió a la producción brasilera, al tiempo que Estados Unidos y Canadá 
iniciaban una tendencia decreciente en el sector ${ }^{4}$. Este comportamiento enseña claras directrices hacia la flexibilización, la producción descentralizada, así como a una mayor concentración del trabajo intelectual en los países de vieja industrialización y la transferencia del trabajo manual hacia regiones en las que se paga menos por la fuerza de trabajo -producto de la valuación monetaria y de las posibilidades de evasión legal.

A su vez, debido a que en el contexto actual, el posicionamiento en el mercado de los productos de la indumentaria depende en gran medida de la penetración de la marca, el trabajo que representa el mayor valor agregado para la empresa es aquél vinculado al diseño, la publicidad y el marketing. En este sentido, lograr un posicionamiento en el mercado requiere que un porcentaje del capital puesto en funciones sea destinado a la publicidad. Este mecanismo estimula la concentración en pocas marcas, en tanto el capital inicial para el despliegue del proceso productivo ha de ser aún más elevado que en otros rubros debido al rol de la imagen de marca en la industria de la moda -la calidad asociada a la durabilidad es un aspecto de segundo orden- (Salgado, 2012).

A nivel local, esta transformación comenzó a llevarse a cabo a partir de la década del 70 y se profundizó promediando la década del 90, cuando debido a la contracción económica, se precipitó el proceso de tercerización de las etapas trabajo-intensivas. Paralelamente las empresas tendieron a la concentración del trabajo intelectual -diseño, publicidad, etc. La consecuente fragmentación del colectivo de trabajadores, agrupados en pequeños talleres, fue uno de los factores que hizo menguar su poder de negociación implicando una notoria reducción tanto del capital variable, como del capital fijo ${ }^{5}$.

4 En el período comprendido entre 1985 y 1995 el promedio de horas semanales trabajadas decreció en África, Asia y las Américas, aumentó en Oceanía y se mantuvo estable en Europa. En 1995 Argentina -junto a Perú- se encontraba entre los países de mayor promedio de horas semanales trabajadas (46-47 horas). Egipto, Corea y Singapur tenían entonces los niveles más altos -58, 51 y 49 horas semanales, respectivamente-. Los países con menor cantidad de horas trabajadas se concentraban en Europa: en el extremo se hallaban Estonia y Dinamarca con 31,5 y 31 horas semanales (datos válidos sólo para los países relevados para el informe de la OIT) (OIT, 2000).

5 No sólo los costos relativos al trabajo fueron transferidos, sino también las maquinarias y los insumos para su funcionamiento pasaron a depender del propietario del taller (Salgado, 2012). 


\section{Deslocalización de las etapas del proceso productivo}

Si reconstruimos el proceso de desintegración vertical de la industria en términos cronológicos, la separación de las unidades ejecutoras de las distintas etapas de producción ocurre antes que su propensión a ser concentradas en diferentes partes del globo. Aquí preferimos exponer la problemática desde la escala mayor en términos geográficos (apartado 1) a la escala menor, atendiendo al carácter particular de la industria de la confección argentina: la mayor parte de su producción se destina al abastecimiento del mercado interno y se encuentra integrada a cadenas globales en muy bajo porcentaje ${ }^{6}$. Es decir, el proceso de relocalización de fases de la producción anteriormente integradas que signa al movimiento de la industria de la confección a nivel mundial, es replicado hacia el interior del perímetro administrativo-político trazado por el Estado-nación. Para comprender el derrotero seguido por la rama, resulta relevante hacer un breve recorrido por su historia.

El despunte de la industria de la indumentaria en Argentina data de fines del siglo XIX. Entre 1870 y 1890 el arribo de trabajadores calificados, así como la introducción de la máquina de coser promovieron el crecimiento del sector (Belini, 2008). De acuerdo al segundo Censo Nacional de 1895 las empresas textiles empleaban mayor número de trabajadores (32.600 personas) $)^{7}$. La tendencia se mantuvo en alza ${ }^{8}$ y se profundizó tras la Primera Guerra Mundial producto de la necesidad de sustituir importaciones. Ya hacia el final de la década la industria de la confección abastecía al mercado interno9. Tras la Segunda Guerra Mundial, la política de industrialización orientada a la construcción de maquinaria dio un nuevo auge a la industria. Entre 1946 y 1952 se dio un impulso a la industria textil mediante políticas proteccionistas, subsidios y créditos. La solidez que adquirió, en cuanto a concentración en tamaño y en empleo, se sostuvo hasta 1975.

Tras la apertura comercial en 1976, comenzó a modificarse el panorama de la industria de la indumentaria -y textil en general-, provocando una caída del 63\% del producto bruto industrial del conjunto de la industria textil en el período comprendido hasta 1981. Las pequeñas y medianas empresas fueron las que recibieron con mayor dureza

6 En materia de exportación el 46\% de las empresas relevadas por la Cámara Industrial Argentina de la Indumentaria (CIAI) en 2013 destinó menos del 5\% de su producción a ventas externas, y el 38\% entre el 5 y el 10\% (CIAI, 2014).

7 El 64\% de los establecimientos industriales se ocupaba en los rubros alimenticio, textil, industrias gráficas y construcción, empleando al $66 \%$ de la mano de obra industrial (Irigoin, 1984).

8 Para 1914, en la industria de la confección se empleaban a 58.000 trabajadores (Belini, 2008) y la producción nacional de productos de vestido y tocador cubría casi el 90\% del consumo interno (Irgoin, 1984).

9 Como producto de la protección aduanera y a la activación del consumo local (Belini, 2008). 
este impacto, lo mismo ocurrió con los trabajadores del sector, cuya ocupación se redujo en un 58\%. La década de los 80 estuvo signada por la retracción debido a la caída de la demanda, ajustes fiscales en un contexto internacional de crecientes barreras arancelarias que implicaron tanto la caída de la producción como de las exportaciones. En este período se cerraron empresas, se redujo la cantidad de trabajadores del sector y las importaciones crecieron en términos relativos.

Con la instalación de la paridad cambiaria en 1991 la industria en general pareció liberarse de las tensiones de la hiperinflación y animarse a proyectar hacia un horizonte fijado en el largo plazo. Sin embargo, no duró mucho la serenidad producto de la apertura económica irrestricta, la revaluación de la moneda local, la escasez de crédito y promoción a la industria, así como la variación en la demanda interna (Adúriz, 2009).

Los primeros signos de retracción comenzaron a avizorarse a partir de 1993. Este fue el punto de inicio de la mayor contracción experimentada por el sector de la indumentaria en 50 años, mayor aún que la de la industria manufacturera en su conjunto ${ }^{10}$. Las empresas apelaron a estrategias de diversa índole a fin de permanecer en el sector, como la fusión de capitales individuales -en algunos casos a través de la concreción de alianzas estratégicas-, reformas hacia el interior del proceso productivo y modificaciones en el circuito de comercialización, fundamentalmente mediante la incorporación de nuevos puntos de venta como outlets, shoppings, venta directa, supermercados, etc. El déficit de la balanza del sector fue en aumento ${ }^{11}$. Según un informe de la Cámara de Indumentaria de Bebés y Niños (CAIBYN) (2010) durante la crisis económica de 2001 la diferencia en el consumo se hizo aún más notoria: mientras el sector experimentaba una caída del $31 \%$, el conjunto del consumo privado agregado se reducía en un $14 \%$. Esto se debe al carácter procíclico de la industria de la confección: en etapas de crecimiento económico y debido al aumento del poder adquisitivo, se incrementa el consumo de indumentaria por encima del promedio de mercancías; a la inversa ocurre en períodos de contracción, dado que la vestimenta suele ser de los primeros productos que se dejan de consumir frente a una reducción del ingreso (Lieutier, 2010).

A raíz de la contracción económica se cerraron gran número de establecimientos y empresas, y se reestructuró la red productiva: desintegración, deslocalización y concentración en las etapas de mayor trabajo intelectual (Salgado, 2010). Para los/as asalariados/as

10 La actividad productiva cayó un 54\%; el empleo, más del 50\%; las horas trabajadas, el $45 \%$ y el salario real, un $20 \%$ (Kosacoff, 2004).

11 Para fines de los años 90 las exportaciones se habían reducido en más del $40 \%$ y las importaciones se incrementaron 50 veces en relación al volumen de importación de fines de la década de los 80 (Adúriz, 2009). 
este proceso implicó despidos masivos debido a una amplia serie de concursos preventivos y quiebras, y sentó las bases para la expansión del trabajo no registrado (Adúriz, 2009).

A partir de 2002 el panorama económico nacional se modificó producto del tipo de cambio alto, debido a la devaluación del peso, sumado a la reactivación de la demanda y a subsidios a los servicios públicos, implicando un crecimiento de la producción nacional. Tal como se ha mencionado, el carácter procíclico de la industria de la indumentaria, hizo posible que fuera uno de los primeros sectores en mostrar una tendencia creciente ${ }^{12}$. Entre mediados de 2002 y fines de 2004 se incrementó la utilización de la capacidad productiva instalada, alcanzando niveles similares a los anteriores a la crisis del $2001^{13}$.

Sin embargo, desde 2004 las empresas comenzaron a depender cada vez más del aumento en el volumen de ventas para compensar el incremento del precio del capital constante y variable debido al efecto de la inflación sobre un tipo de cambio que se mantuvo estable. A partir de 2005 el sector mostró una considerable desaceleración del crecimiento ${ }^{14}$ : fundamentalmente debido al raudo incremento de las importaciones producto de la apreciación relativa de la moneda en términos reales, en 2007 llegaron a superar los niveles máximos alcanzados en la década del $90^{15}$.

La conjunción del estallido de la crisis económica mundial con el crecimiento de las importaciones a precios muy inferiores a los de producción local, hizo que la caída de la demanda interna precipitara la detención del crecimiento. Si bien desde 2005 el gobierno fue aplicando mayores exigencias a los productos importados, recién a partir de mediados de 2008, frente a los primeros signos de retracción, las medidas cobraron gran importancia. Aun así, el incremento de las importaciones ha profundizado la contracción del sector (CAIBYN, 2010).

De acuerdo al análisis comparativo de precios de importación de indumentaria realizado por la CAIBYN en 2010, se destaca la gran diferencia existente entre el de los productos provenientes de China, 13,40 dólares por kilo, y el precio promedio de todos

12 Mientras el nivel general del sector secundario de la economía en el primer trimestre de 2003 aumentó un 19\%, el sector confeccionista se incrementó en un 63\% (CAIBYN, 2010).

13 Llegó a alcanzar un nivel de utilización de la capacidad instalada del $74 \%$ en el 2003 , incorporando 72 mil nuevos trabajadores/as en forma directa en un año y medio (Adúriz, 2009).

14 Mientras que entre 2002 y 2005 la tasa de expansión acumulada había sido de 74,6\%, entre 2005 y el primer cuatrimestre de 2008 sólo representó el 17,7\% (CAIBYN, 2010).

15 Entre 2002 y 2007 la tasa promedio anual de crecimiento de la producción nacional fue del 17\%, al tiempo que la de las importaciones, en el mismo lapso, fue del 36\% (Adúriz, 2009). 
los orígenes 25,10 dólares. La industria local produce a un promedio de 24,50 dólares por kilo, ubicándose también muy por encima del precio de venta chino. Concretamente en lo que refiere al ámbito de la indumentaria, en Argentina, la presencia de productos chinos aumentó del 6\% en 2003 al 57\% en $2008^{16}$. Según información publicada por la Cámara Industrial Argentina de la Indumentaria (CIAI) (2014) este precio es alcanzado debido a la existencia de dumping. De acuerdo con la información publicada, el margen de dumping ${ }^{17}$ determinado asciende al $561 \%$ en $2010^{18}$.

La apertura comercial de China a partir de 2001 -año de su incorporación a la Organización Mundial del Comercio-, así como la eliminación a las restricciones en la Unión Europea y los Estados Unidos (Tras la Etiqueta, 2008), junto a la finalización del último de los acuerdos que intervinieron en la regulación de la cadena desde mediados del siglo XX en 2005, abonaron al posicionamiento privilegiado de la indumentaria china en el mercado mundial. Asimismo, las condiciones en que se produce en este país son explicativas de los bajos precios de venta de las mercancías. A partir de la apertura comercial se inició una gran migración del campo a la ciudad. La incorporación de los/as trabajadores/as -anteriormente campesinos-as- al trabajo en las grandes urbes acarreó limitaciones para esta población en cuanto al acceso a la protección estatal debido a lo dispuesto por el Sistema Hukou ${ }^{19}$, implicando restricciones similares a las de la migración indocumentada. Según datos de 2004 publicados por el Bureau Nacional de Estadísticas, el ingreso promedio mensual de los migrantes internos se acerca a la mitad del pagado a la población urbana. La jornada laboral se extiende en promedio a 11 horas y se trabajan 26 días al mes. Sólo alrededor del 10\% de los migrantes cuentan con un contrato de trabajo y un seguro de salud. En los trabajos a los que acceden, las condiciones de seguridad e higiene no están garantizadas implicando en muchos casos graves consecuencias para la salud. En las ciudades con gran concentración de población migrante existen enclaves conocidos como villas urbanas (chengzhongcun) en donde habitan en condiciones de hacinamiento (China Labour Bulletin, 2013).

16 Los porcentajes refieren al peso de los productos (CAIBYN, 2010).

17 El margen de dumping se establece a partir de la diferencia entre el valor normal -precio comparable de un producto similar destinado al consumo en el país exportador- y el precio de exportación.

18 Se trata específicamente de trajes para hombres o niños, conjuntos y chaquetas. La investigación fue realizada por la Dirección de Competencia Desleal de Argentina (China.org, 2010).

19 Consiste en un sistema de permisos de residencia en China que limita la posibilidad de emigración a otro lugar. Fue instaurado en 1958 con el fin de controlar el éxodo a las grandes urbes. El sistema de Hukou en China divide a la población en dos clases: hogares urbanos y hogares rurales y prevé el acceso a la seguridad social, educación, salud y al mercado laboral acorde al lugar de registro. Es decir, sólo en el marco de las fronteras administrativas del lugar de residencia todos los derechos y beneficios están garantizados. Para 2014 sólo el 36\% de las personas que viven en las ciudades califican como residentes (The Economist, 2014). 
El empleo de esta población en las condiciones mencionadas supone una reducción del capital variable que se traduce en menores precios de producción y venta de las mercancías. Los bajos precios de venta de los productos chinos han dado aliento al aumento del plusvalor absoluto, incremento de la extensión de la jornada de trabajo, y de la precarización de las condiciones de trabajo y empleo para permanecer en el mercado. Esto se ha llevado a cabo a través de la tercerización a talleres de confección clandestinos. Según se sostiene en el Informe sobre el Sector de la Indumentaria en Argentina publicado por el Instituto Nacional de Educación Tecnológica en 2008:

la introducción de mano de obra barata de países pobres de la región -Bolivia en este caso- se ha convertido en la alternativa más viable para las empresas y marcas que trabajan con talleres, reduciendo las remuneraciones promedio del sector y desplazando la mano de obra local (p. 104).

En otros términos, se puede afirmar que la alternativa desplegada por el capital local para permanecer en el sector ha sido, en definitiva, la importación de las relaciones de producción.

En síntesis, más allá del crecimiento experimentado a partir de 2002, la producción de indumentaria no ha recuperado la participación que tenía en el conjunto de la industria nacional antes de la década del 90 . Un fuerte proceso de transformación viene operándose al interior del proceso productivo. La reducción de costos de capital variable y fijo ha sido la estrategia desplegada por muchos, a través de la creación y proliferación de pequeños talleres a los que tercerizar la confección, asentando gran parte de la producción en un trabajo altamente precarizado.

\section{Deslocalización de la fuerza de trabajo}

La reestructuración capitalista que se inició a partir de la crisis de mediados de los 70 signó la coyuntura sobre la que se iba a emplazar la desarticulación de la creciente homogeneidad de la clase obrera que había sustentado un largo ciclo de luchas. Entonces, en términos de organización industrial, la fractura del proceso de producción y su deslocalización se tradujeron en la fragmentación del colectivo de trabajadores/as. A este respecto, Altamira (2001) señala que en esta transformación: 
los grandes consensos keynesianos, responsables de la integración social, fueron sustituidos por la ruptura de la unidad de clase, fractura interna que se expresa en el avance de las negociaciones individuales y en la creciente disminución de la sindicalización y en la constitución de una nueva minoría, los parados (p. 108).

El dinamismo que fue adquiriendo la producción, requirió crecientemente de una fuerza de trabajo flexible y polivalente, e incluso -como veremos más adelante- dispuesta a desplazarse en el espacio.

\section{El trabajo a domicilio: lo nuevo de la tercerización siempre presente}

El trabajo a domicilio es una de las formas características que asume la relación laboral en el sector de la indumentaria. Esta da cuenta de una conformación de la cadena de valor donde la tercerización siempre ha sido parte constitutiva. Sin embargo, como se verá, este formato fue precarizado en línea con las transformaciones operadas en el mundo del trabajo a partir de la reestructuración capitalista. En este apartado nos proponemos analizar qué es considerado dentro de esa nominación, específicamente en cuanto a su uso legal, así como aproximarnos a su historia en la industria de confección.

Tradicionalmente el trabajo a domicilio se ha concentrado en los sectores de mayor intensidad, relacionado a situaciones de sobre-explotación, precariedad y pobreza ${ }^{20}$. Los límites que circunscriben a este tipo de trabajo resultan difusos, principalmente en torno a su distinción del trabajo autónomo. Argentina cuenta con una legislación sumamente progresiva en esta materia: la Ley 12.713 de Trabajo a Domicilio fue promulgada en 1941, para refrenar la sobreexplotación de estos trabajadores. Esta ley considera al dueño del taller en una doble figura: empleador de los/as costureros/as y empleado de las marcas que encargan y compran su producción. Al momento de su sanción se procuró que los/as trabajadores/as empleados/as en talleres contaran con un respaldo legal similar al que tenían los/as empleados/as en fábricas. Es notorio que, a diferencia de lo estipulado habitualmente por la legislación laboral, esta ley incluya artículos penales para quienes paguen por debajo de los salarios acordados. La Ley de Trabajo a Domicilio

20 Si bien en la actualidad esa continúa siendo la realidad para la mayor parte de los/as trabajadores-as a domicilio, también se ha comenzado a extender a tareas calificadas -vinculadas a la tecnología, a procesos administrativos, etc.- como forma específica del teletrabajo, por las que se pagan salarios más elevados y cuentan con mejores condiciones laborales que aquéllas que utilizan mando de obra intensiva (Abramo, Rodríguez y Rossignoti, 2004). 
resulta auspiciosa en términos del reconocimiento de la relación de dependencia con las grandes marcas, dado que al considerar al tallerista como empleado de éstas, establece una relación directa entre la marca que contrata el trabajo y los/as trabajadores/as del taller $^{21}$ (Salgado, 2011). Sin embargo, la aplicación y reglamentación de la ley ha sido llevada por un derrotero que dio por resultado una capacidad de protección de los/as trabajadores/as tan baja como las propias de países con legislaciones más rezagadas ${ }^{22}$.

En 1996 la OIT adoptó el convenio 177 a fin de unificar criterios para su demarcación, cuyos puntos básicos fueron:

- el trabajo se realiza en locales que no pertenecen al empleador (el propio domicilio u otros);

- el trabajador percibe una remuneración por el trabajo realizado;

- el empleador dispone las especificaciones del trabajo, independientemente de la provisión de maquinarias, materias primas, etc., utilizadas para la producción "a menos que esa persona tenga el grado de autonomía y de independencia económica necesario para ser considerada como trabajador independiente en virtud de la legislación nacional o decisiones judiciales" (Jelin, Mercado y Wyczkier, 1998, p. 3).

Las vastas zonas grises que han de interpretarse acorde a las leyes de cada país y a su jurisprudencia se vinculan con el establecimiento de la subordinación del trabajador, expresadas en los siguientes factores:

- utilización de maquinaria propia;

21 En 2008 se intentó modificar la Ley procurando romper el lazo entre el taller y la empresa mediante la constitución del primero en PyME (pequeña y mediana empresa). En el proyecto de modificación se proponía eliminar la figura del tallerista, modificar la forma de solidaridad y suprimir los artículos penales. Esta reforma alteraría el espíritu de la Ley al trasladar la responsabilidad sobre los/as trabajadores/as al tallerista, para desvincular a las grandes empresas. Producto de la movilización de diferentes organizaciones sociales en contra de la modificación, entonces no se avanzó con la misma.

22 Jelin, Mercado y Wyczykier (1998) analizan en detalle su aplicación a lo largo del tiempo, así como los cambios de figuras institucionales con facultad de policiamiento del trabajo a domicilio. 
- contratación de fuerza de trabajo auxiliar por parte de quien recibe trabajo;

- control supervisado de la producción.

Existen formas que se circunscriben en lo denominado fraude laboral que confunden y abonan al posicionamiento del trabajador a domicilio como trabajador autónomo ${ }^{23}$. Sin embargo, lo que no hay que perder de vista para analizar la relación de dependencia, es el grado de autonomía que los trabajadores tienen en la definición de sus tareas: el control de la producción, la supervisión del trabajo -in situ o no-, el establecimiento de plazos, los compromisos a que se somete al trabajador, la asiduidad del vínculo, etc., son factores que determinan la existencia de una relación laboral.

Otro aspecto que contribuye al maremágnum en la definición del tipo de lazo laboral, remite a la registración en doble sentido: laboral e impositiva. Gran cantidad de talleres se halla en la clandestinidad en cuanto a la carencia de registro del establecimiento y de los trabajadores. Si bien, en Argentina los registros oficiales de esta modalidad de trabajo son escasos -como ocurre en América Latina en general, producto de limitaciones en las definiciones y de la inexactitud de los instrumentos de medición ${ }^{24}$, se estima que el subregistro del trabajo a domicilio es de una cuantía considerable y se relaciona con la falta de registro del taller, la ilegalidad de la relación laboral y las imprecisiones en la definición quienes trabajan bajo esta modalidad (Jelin, Mercado y Wyczykier, 1998) ${ }^{25}$. Los datos del Archivo de Inscripciones del Departamento de Trabajo a Domicilio ${ }^{26}$ muestran que un gran porcentaje de los trabajadores a domicilio se concentra en el sector de la confección de indumentaria. Desde 1950 hasta $1996^{27}$ las inscripciones han seguido una tendencia decreciente (Jelin, Mercado y Wyczykier, 1998), lo cual no se explica por la reducción de este tipo de trabajo, sino por la expansión de la clandestinidad como se expresa en diversos informes basados en análisis cualitativos (Gallart, 2006; OIT-CEA, 2011).

23 Ejemplos de esto son: el pago de honorarios en lugar de salarios, la exigencia de su inscripción como autónomos bajo el régimen de monotributo, la contratación por obra, etc.

24 Los criterios e instrumentos estadísticos utilizados en la región logran en general captar el trabajo en el domicilio, pero sin poder distinguirlo claramente del trabajador autónomo (Abramo, Rodríguez, y Rossignotti, 2004).

25 De acuerdo a datos del censo del año 80, el 80,5\% de los trabajadores domiciliarios que trabajaba en la confección de indumentaria en el Gran Buenos Aires declaraba ser independiente, $4 \%$ se definía como patrón o socio y sólo el 8,8\% se consideraba asalariado (Marshall, 1990).

26 El archivo dependía del Ministerio de Trabajo y Seguridad Social (Jelin, Mercado y Wyczykier, 1998) hasta que sus competencias fueron transferidas. En la actualidad no existe un archivo unificado.

27 Con la excepción del año 1992 en que hubo un fuerte operativo de inspección que se tradujo en un mayor número de inscripciones. 
Entre las características que asume el trabajo a domicilio hay que destacar la forma más extendida de remuneración: el pago a destajo. La OIT estima que esta modalidad se replica en todo el mundo y los montos que se pagan suelen ser inferiores que los de trabajadores fabriles (OIT, 1996). En la jerga del sector, el trabajo a destajo se denomina trabajo a fasón, el uso extendido de esta nominación da cuenta de la naturalización de este tipo de pago por la fuerza de trabajo.

Si bien en la actualidad es difícil estimar el porcentaje de mujeres que trabajan en la confección, debido al alto nivel de no registro, históricamente la rama se ha caracterizado por una gran presencia femenina ${ }^{28}$ ya que el trabajo en el hogar permite, en algún modo, la combinación de las actividades remuneradas con las domésticas, endilgadas tradicionalmente al género femenino, y puede representar en ocasiones la única alternativa para la percepción de un salario. Tomei (1999) sostiene que en muchos casos el rendimiento se aumenta mediante el trabajo familiar, el cual es vivenciado como una ayuda o una suerte de extensión de las responsabilidades domésticas, fundamentalmente de hijos/as. Además, la apariencia de cuentapropismo que reviste el trabajo a domicilio es utilizada para hacer recaer sobre los/as trabajadores/as la responsabilidad por fallas y errores.

La invisibilidad del trabajo a domicilio se expresa tanto en materia laboral, estadística, como en términos sociopolíticos (Abramo, Rodríguez y Rossignotti, 2004). Diferentes factores confluyen en dejar a los trabajadores desprovistos de una adecuada protección laboral y social: el carácter mayormente informal de los establecimientos productivos, la exigua aplicación de la ley, la desidia de las instituciones dedicadas a su control, a lo que se suma el aislamiento, producto de la atomización, que restringe la posibilidad de conformación de un colectivo capaz de reclamar el respeto a la ley y la mejora de las condiciones.

Por otro lado, la multiplicidad de eslabones presentes en el proceso productivo, como veremos a continuación, abona a la dilución de la figura del patrón desorientando en torno a la concernencia de las responsabilidades legales.

${ }_{28}$ Según datos del censo de 1980 el 82\% eran mujeres. 


\section{Transformaciones en la cadena de valor y fragmentación del colectivo de trabajadores/as}

Este apartado lo iniciamos presentando la idea de Lebowitz (2005) acerca de la centralidad de la lucha de clases en la determinación de la expansión del capital, como cardinal para el abordaje del caso en estudio. La forma que asume el trabajo en la industria de la confección actual ilustra esta tesis en el sentido contrario: allí donde la organización de clase está desarticulada, el capital se expande hasta el máximo tolerable por el cuerpo humano. El autor retoma a Marx para reflexionar en torno a la capacidad de asociación de los/as trabajadores/as en la industria domiciliaria y en la manufactura: la disgregación presente en la primera es a través de lo que se explica la escasa capacidad de resistencia en comparación con la segunda. En virtud de esta diferencia Lebowitz sostiene que

la magnitud del plusvalor extraído por quienes median entre los productores (o sea, la medida de la explotación) depende del grado de separación de los productores y concluye que sólo a través de la lucha por reducir su grado de separación, los trabajadores serán capaces de lograr sus objetivos (2005, p. 135).

Utilizando esta reflexión como vector, analizaremos las particularidades del proceso productivo en el presente para comprender de qué modo la extensión del trabajo a domicilio se relaciona con el crecimiento de la clandestinidad y el empleo de migrantes indocumentados.

A pesar del crecimiento que viene mostrando el sector desde la última década, aún no alcanza los niveles anteriores a los años 90. Si bien, formas características, como el trabajo a domicilio y el pago a destajo, sostienen su protagonismo, la desintegración vertical del proceso productivo, como hemos señalado, implicó la generación de nuevos encadenamientos entre los eslabones tradicionales del sector. Proceso que fue acompañado por la atomización de las actividades trabajo-intensivas.

Cada una de estas instancias del proceso de producción tiene una fuerza relativa diferencial vinculada tanto a su posición estratégica en la cadena de valor, como a su capacidad organizativa con sus pares y de ello deviene una consecuente apropiación del plusvalor.

Una primera línea divisoria en la cadena de valor de indumentaria es la que separa el trabajo intelectual y el manual. El primero involucra a las tareas relativas a la definición 
y diseño del producto, organización de las tareas, confección de moldes, así como a las referentes a la gestión de los medios de producción. El trabajo manual comprende las tareas de costura, estampa, cuellos, ojales, etc. El corte es la única tarea manual que habitualmente se lleva a cabo en el mismo establecimiento en que tienen lugar labores asociadas al trabajo intelectual debido a la especialización que requiere y a las pérdidas de materia prima que representan los errores en esta instancia, como veremos a continuación.

En la actualidad la cadena de valor de indumentaria ha crecido en intermediaciones como resultado de la desintegración vertical del proceso de producción. Haciendo una vaga abstracción del conjunto de instancias involucradas en la producción de indumentaria podemos identificar una patronal dividida en tres grandes grupos: fabricantes, intermediarios y talleristas. A continuación analizaremos sucintamente las tareas que llevan a cabo, así como las características que le confieren un determinado poder de negociación.

1. Fabricantes: Un variado gradiente es comprendido en esta categoría. En un extremo se encuentran las fábricas con alta integración vertical y cuya existencia suele ser anterior a la década del $80^{29}$. Éstas llevan a cabo todas las tareas previas a la comercialización: diseño, corte y confección ${ }^{30}$; y tienen la mayor parte de su personal registrado. Algunas de ellas sólo mantienen su producción, pero no se amplían. Existen también pequeños fabricantes que tercerizan la tarea de confección a pequeños talleres. Y en otro extremo encontramos a los diseñadores de alta costura y fabricantes virtuales ${ }^{31}$ que se ocupan del posicionamiento en el mercado y el diseño, parte considerable del capital es invertido en estas instancias, y tercerizan la confección; sus productos se orientan usualmente a segmentos de alto poder adquisitivo lo que posibilita tener elevados precios de venta. A diferencia del fabricante tradicional, en este tipo de emprendimientos surgidos en las últimas décadas, la costura es tercerizada (Gallart, 2006).

En diferentes medidas según sus características, este conjunto -que comprende a la mayor parte de la patronal- es el que determina las características de la producción,

29 Las tradicionales empresas productoras de indumentaria, que integraban la totalidad del proceso productivo en un mismo establecimiento han desaparecido casi por completo en la actualidad. Lo único que subsiste en algunos casos es la tradición de algunas marcas en el sector, sustentada en parte por una trayectoria familiar que se ha ocupado del sostenimiento de la marca-fábrica (OIT-CEA, 2011).

30 En el ámbito registrado se han incrementado las empresas micro y pequeñas mientras que las grandes y medianas se redujeron proporcionalmente (OEDE - MTySS).

31 También denominados Fabricantes sin fábricas o Marqueros. 
diseño gestión de la materia prima, mantenimiento de la relación con talleres de confección y puntos de venta, etc., impone los tiempos y se apropia de aproximadamente un $20 \%$ del precio de venta minorista. Las dificultades que manifiestan refieren fundamentalmente a los precios de las materias primas y a la implementación de medidas proteccionistas que permitan menguar la competencia externa.

2. Recolector o intermediario tallerista: Se ocupa de las etapas de organización de la producción relacionadas con la intermediación entre las empresas confeccionistas y los trabajadores a domicilio. Es decir, interviene en la contratación y control del trabajo, pero no es el propietario de la mercancía. Cuenta con información y contactos tanto de proveedores e insumos, como de especialistas -moldería, estampería, diseño, etc.- y de talleres de diversas dimensiones (Gallart, 2006). Los recolectores se apropian de un monto similar o mayor al de las empresas confeccionistas. Cuando éstos no intervienen, ese rol es llevado a cabo por el fabricante.

3. Talleristas (costura, estampa, cuellos, ojales): Existen talleres registrados que cumplen con la normativa laboral e impositiva y trabajan en general para una marca o un pequeño conjunto de ellas, con exclusividad. Luego se encuentra un amplio gradiente de talleres carentes de registro poseedores de unas pocas máquinas acorde a los testimonios, raras veces superan las 40-. En éstos trabajan mayormente personas procedentes de Bolivia. Aquéllos que son más grandes suelen reclutar a los/as trabajadores en el país de origen y traerlos clandestinamente, inscribiendo este mecanismo en la trata de personas ${ }^{32}$. Los talleres más pequeños, en general, corresponden al trabajo familiar ${ }^{33}$.

En muchos casos, fundamentalmente en los establecimientos más precarios y donde se trabajan más horas, las condiciones de seguridad e higiene no están garantizadas. Enfermedades como tuberculosis se han presentado con frecuencia entre las personas que trabajan en talleres clandestinos debido a la permanente inhalación de polvo y polvillo, a las condiciones de hacinamiento en que viven y trabajan, y al debilitamiento

32 En el informe de la OIT- CEA (2011) se propone una clasificación alternativa a la desarrollada por el Instituto Nacional de Tecnología Industrial (INTI) en 2005 -talleres integrales, especializados y secundarios- debido a que esta resulta insuficiente para reflejar la dispersión de establecimientos productivos y la consecuente movilidad que esto genera entre las categorías establecidas por éste organismo: dependiendo de las condiciones de producción y entrega que sean negociadas, un mismo taller puede ocupar un rol especializado o secundario, así como operar de receptor o dador de trabajo. Alternativamente la OIT-CEA (2011) propone categorías que dependen del tipo y la cantidad de máquinas, y del tipo de trabajo encomendado -algunas prendas son confeccionadas en diversos talleres acorde a su especialidad-.

33 Categoría definida como obrero por la Ley 12.713 de Trabajo a Domicilio. 
del sistema inmunológico producto de la mala alimentación y la fatiga que suponen las largas jornadas laborales (Goldberg, 2010).

Los talleres suelen caracterizarse por su invisibilidad, se procura que no sean distinguibles en el paisaje urbano como un establecimiento productivo, motivo por el que se dificulta su detección. A esto se suma la irregularidad, en que se halla la mayoría, en materia laboral e impositiva. Sólo en algunos casos, las mercancías se registran mediante facturas provistas por el tallerista inscripto en el régimen de monotributo. Las instancias de registro se ubican habitualmente en la compra de la materia prima, el diseño, el tizado y el corte, luego de estas etapas el proceso puede seguir un itinerario en la clandestinidad para la confección de las prendas. Cuando el producto terminado es ingresado al circuito de la comercialización, vuelve a formalizarse (Gallart, 2006), exceptuando los casos en que se comercializa en el circuito informal.

La dispersión de los talleres, el escaso número de personas involucradas en la producción y, fundamentalmente, la situación de clandestinidad en que se encuentran muchos de ellos, comporta una gran debilidad de negociación en relación a las marcas.

A esto se adiciona, el aceleramiento de los tiempos de producción que impone el ritmo de la moda lo que trae aparejado un desorden en la planificación del proceso productivo. Esta inestabilidad de los pedidos vulnera la estabilidad laboral al generar una absorción de trabajo por encima de la capacidad productiva en temporada alta, provocando la extensión de las jornadas, y el cierre de establecimientos de producción en épocas en que se reducen los pedidos. Producto de este amplio conjunto de factores se encuentran condiciones de alta precarización hacia el final de la cadena de valor.

En cuanto al colectivo de trabajadores se puede establecer una primera línea divisoria relacionada con el carácter de cada labor como proponemos a continuación:

1. Cortadores: Ocupan un lugar central en el proceso productivo debido a que las telas se cortan encimadas, por lo que la falta de precisión o un error llevan a la pérdida de la materia prima. Debido a esto, los moldes y el tizado -el dibujo del modelo en la tela encimada- es considerado un trabajo de importancia superlativa. El tiempo de aprendizaje de esta actividad es extenso, lo que supone una especialización que vuelve a este trabajo clave y su demanda en el sector es permanente.

2. Costureros/as y bordadores/as: Son quienes se ocupan de la última fase del proceso de productivo: la confección. Incluso quienes están registrados tienen ingresos 
sensiblemente inferiores a otras ocupaciones. Pueden desempeñarse en talleres formales, informales o trabajar desde su domicilio, caso en el que los errores de confección recaen sobre el trabajador. Su situación es de gran precariedad, no sólo por lo magro de sus ingresos, sino también por la casi inexistente cobertura de derechos sociales.

La situación de aislamiento -carencia de relaciones con otros trabajadores- y dependencia de quien contrata su trabajo, van en detrimento de sus posibilidades de negociación. De esta manera resulta evidente la forma en que la presión de la producción recae sobre el/la trabajador/a, quien se posterga a fin de cumplir con las exigencias del patrón en desmedro no sólo de su tranquilidad, sino también de sus ingresos.

Las implicancias de esta división del trabajo entre quienes intervienen en el proceso de producción de indumentaria saltan a la vista al analizar el reparto del plusvalor: mientras el fabricante retiene aproximadamente el $20 \%$, en la comercialización se apropia un $29 \%$, el taller registrado sólo percibe un $2,9 \%$ del precio de venta minorista de la prenda, en el caso de los talleres clandestinos dicho porcentaje se estima en un 1,3\%. El/ la costurero/a recibe apenas un $1,8 \%$ de dicho precio en concepto de salario (Lieutier, 2010).

La dinámica imperante en esta forma de distribución de la producción y su extensión en el tiempo han generado una trama sólida en la que el trabajo no registrado comienza a aparecer como una condición indispensable para la reproducción del capital en este ámbito, a punto tal que la evasión legal no sea percibida como una amenaza ${ }^{34}$.

La concentración diferencial de capital en el sector de la indumentaria habilita a los fabricantes a fijar los precios a los talleres de confección (Salgado, 2012). Según Lieutier (2010), su atomización y falta de organización los compele a aceptar los precios impuestos como única alternativa para colocar su producción y concluye que "de esta manera, el fabricante explota al tallerista y, en ese mismo acto, convierte al tallerista en explotador de los costureros" (p. 102). Si bien talleristas y costureros se hallan en uno de los extremos de la cadena de valor y cuentan con las peores condiciones de negociación, consideramos fundamental pensar críticamente la decisión de explotar a otro/a. Es decir, valerse de una mirada múltiple sobre el caso capaz de captar la relación capital-trabajo con diversos puntos de fuga: tanto hacia el taller, como a través de toda la cadena de valor. Este resulta, a la vez, un ejercicio de vigilancia epistemológica en la medida que permite

34 Según Gallart (2006) la antigüedad del emprendimiento, su tamaño y la clientela objetivo son los factores de mayor incidencia a la hora de optar por el trabajo no registrado. El último resulta de singular relevancia en tanto se relaciona con los precios de venta, el volumen y la frecuencia de producción. 
reflexionar en torno a la relación entre la agencia y las determinaciones económicas, étnicas, familiares, de género, etc. Si bien, los escasos márgenes que deja la actividad, incluso extendiendo la carga horaria hasta el máximo tolerable, juegan en favor de hacer recaer ese esfuerzo sobre otros hombros, no todos/as los-as costureros/as se hacen talleristas $y$ esto nos da la pauta de que no se trata de la única dirección posible.

Así retorna la idea de pérdida de fuerza y de derechos para un colectivo de trabajadores/as fragmentado. Lebowitz (2005) retoma lo expuesto por Engels (1881) en un artículo publicado por The Labour Standard sobre sindicatos. Allí el autor sostenía que la negociación sin la organización de los/as trabajadores/as implica una creciente tendencia a la baja salarial y al aumento de las horas de trabajo. De este modo, superar la desunión de los/as trabajadores/as "como vendedores de mercancías que compiten entre sí” (Lebowitz, 2005, p. 138) se impone como necesidad primera para accionar contra esta tendencia.

\section{Trabajadores/as migrantes en la producción de indumentaria argentina}

Como adelantábamos más arriba la migración enseña un crecimiento acelerado desde la última reestructuración capitalista. Las disparidades de poder adquisitivo entre los Estados-nación, así como en materia de conflictividad en un mundo en creciente integración productiva y cultural, han ido impulsando el desplazamiento de vastos sectores de población como alternativa para la mejoría de las condiciones de vida. La proliferación de los desplazamientos internacionales implicó una variedad de migraciones indocumentadas y de configuraciones de la ilegalidad. De Génova (2002) encuentra en la extensa bibliografía sobre el tema que las migraciones indocumentadas se sustentan preeminentemente en motivos laborales. Este carácter resulta central para analizar el caso en estudio.

Asimismo, como advierte Sassen (2007), las migraciones no se hacen solas, son producidas e involucran una combinación posible de países. En este apartado desarrollaremos la historia de desplazamientos desde Bolivia a Argentina a fin de analizar la conformación de una dirección, un canal preferente, un puente que promueve ciertos destinos y no otros. 
La migración de países limítrofes es un fenómeno cuya historia se remonta a la conformación del Estado nacional. Si bien, en la actualidad posee gran visibilidad, numerosa cantidad de estudios dan cuenta de la perennidad de su existencia, así como de su variabilidad. Hacia finales del siglo XIX representaban un bajo porcentaje en relación al conjunto de inmigrantes (Germani, 2010). Esta relación fue decreciendo hasta 1914, año en el que llegó al 8\%, a partir de entonces inició una tendencia ascendente: para 1991 representaba a la mitad del conjunto de inmigrantes y en 2001 alcanzó el 60\%. Dado que en la industria de la confección se emplea mayormente a migrantes procedentes de Bolivia (INET, 2008), haremos foco en la migración desde este país para analizar posteriormente su relación con el devenir de la industria de la confección.

Las primeras migraciones provenientes de Bolivia tenían un carácter rural-rural: se ocupaban de cubrir la demanda de trabajo estacional propia del trabajo agrícola concentrándose fundamentalmente en Salta y Jujuy ${ }^{35}$. Tras el impulso de los cultivos de caña de azúcar no sólo se incrementó el flujo migratorio, sino también muchos comenzaron a establecerse en Argentina ${ }^{36}$. Esta tendencia se intensificó hacia los inicios de la década del 40: los inmigrantes combinaban diferentes trabajos estacionales vinculados a la frutihorticultura. Según estimaciones en ese entonces casi el 90\% se establecía en el norte, en tanto un 7\% se asentaba en Buenos Aires. Promediando el siglo XX la migración comienza a adoptar una naturaleza rural-urbana. Las ocupaciones que concentraban la mayor cantidad de inmigrantes bolivianos en el Gran Buenos Aires (GBA) ${ }^{37}$ durante la década del 60 tenían como denominador común la baja calificación y la inestabilidad: trabajo doméstico, industria de la confección, construcción, horticultura, venta callejera de verduras (Pascucci, 2010). Según Benencia (2009). La atracción hacia las metrópolis era provocada por la posibilidad de conseguir mejores salarios que en el país de origen o en las zonas fronterizas.

En 1970 más de un tercio de los inmigrantes de países limítrofes habitaba en el área metropolitana de Buenos Aires. Este acrecentamiento de su presencia en centros urbanos otorgó mayor visibilidad al fenómeno. Para Caggiano (2005) este cambio se debió a la expulsión de la mano de obra estacional de las actividades vinculadas a la agricultura producto de su tecnificación, así como a las potencialidades de mejora del estándar de vida que se vincula a los centros urbanos.

\footnotetext{
Concluyendo el siglo XIX, la mayor parte de los inmigrantes provenían de zonas empobrecidas del Altiplano.

Se estima que en mayo de 1930 más de 30.000 personas ingresaron al país.

37 Para entonces el GBA era el lugar de destino del $45 \%$ de los inmigrantes bolivianos, así como de argentinos provenientes de diferentes provincias.
} 
Hacia 1991 el 40\% de los migrantes de Bolivia se asentaba en la ciudad de Buenos Aires y el GBA. De acuerdo a datos del censo 2001, la proporción de migrantes bolivianos en el área metropolitana del GBA mantiene una tendencia creciente de $51,6 \%$, en tanto se reduce la de aquellos que residen en Salta y Jujuy al 23,8\%. La crisis de 2001-2002 operó como punto de inflexión en materia de empleo de migrantes en la industria de la confección. La retracción económica llevó a la reubicación en industrias más débiles y de alta precarización -vestido, calzado y comercio al por menor, fundamentalmente-. El 35\% de los migrantes provenientes de Bolivia, Paraguay y Perú se concentran en los barrios de Villa Lugano, Flores, Nueva Pompeya y Balvanera; mientras que en el GBA el 20\% se ubica en La Matanza y el 9\% en Lomas de Zamora (Cerrutti, 2009). Estas zonas coinciden con las de mayor concentración de talleres dedicados a la confección de indumentaria, actividad en la que se desempeña gran parte de las personas que proceden de Bolivia (Pascucci, 2010).

A partir de los datos cualitativos y en línea con otros estudios académicos se evidencia que quienes llegan a trabajar al ámbito de confección de indumentaria provienen de hogares de bajos recursos, en muchos casos se trata de una estrategia familiar por etapas, en otros, el migrante se acerca a parientes menos cercanos que ya están asentados en la ciudad de destino. El tipo de trabajo al que acceden es de baja calificación, se encuentra en el límite inferior de la escala salarial y se asocia a una alta rotación (incorporación y expulsión veloz de trabajadores del proceso productivo).

La trata de personas con fines de explotación laboral se ha ido extendiendo en el tiempo y explica parte de la migración de países limítrofes ${ }^{38}$. En el sector textil, específicamente, prevalece la modalidad definida como servidumbre por deudas. Generalmente, la deuda inicial consiste en los gastos de traslado desde el país de origen, ésta es cobrada con trabajo en el lugar de destino. Dado que la vivienda y la comida son provistas por el empleador, esta deuda se va acrecentando y debe ser saldada con un trabajo por el que se paga un salario muy inferior al estipulado por la legislación labora ${ }^{39}$.

El sometimiento y las obligaciones son generados también a través de mecanismos como la retención de los documentos de identidad, el pago retrasado del sueldo y en pequeñas partes, las amenazas con la puesta en conocimiento a las autoridades de

38 En el caso que analizamos, implica también el tráfico, debido a que son trasladadas a otro país de manera irregular. Se considera un delito contra las personas que se caracteriza por un reclutamiento a través de engaños, rapto o coacción cuya finalidad es la explotación laboral, sexual o la extracción de órganos (D’Ovidio, 2007).

39 En Argentina se estiman entre 100.000 y 130.000 las víctimas de trata con fines de explotación sexual y laboral provenientes de Bolivia (D’Ovidio, 2007). 
migración, la prohibición de entrar y salir del taller libremente, para mencionar los que se han denunciado con mayor frecuencia. Asimismo existen denuncias de violencia física, apropiación de las pertenencias del trabajador por parte del tallerista y abuso sexual. En diversos testimonios se da cuenta de la utilización de la posibilidad, ilusoria, de la deportación por parte de talleristas para generar obediencia de parte de los/as costureros/as.

Cómo salir del circuito de la costura, es un interrogante que requiere la mayor atención. En muchos casos el ascenso se proyecta hacia la figura de tallerista. Tras varios años de residencia, algunos costureros/as han constituido talleres familiares, al menos en los inicios, reproduciendo las condiciones de explotación a las que fueron sometidos. Algunos/as encuentran otras actividades en las que también existe gran presencia de trabajadores/as migrantes y una elevada precarización del trabajo y el empleo, aunque menor que en la confección. Fundamentalmente la construcción y el trabajo en casas particulares. En el presente las actividades en que se desempeñan los y las migrantes de Bolivia de primera generación se reducen a un escaso número. Todavía se requiere de un gran trabajo para impedir que se haga usufructo de la situación de vulnerabilidad en que se hallan estas personas.

\section{Conclusiones}

Hemos analizado el modo en que se vinculan la extensión de condiciones de trabajo altamente precarizadas con las transformaciones del capital a escala planetaria, incorporando la dimensión geográfica como sugiere Harvey (2007) para el análisis de la relación capital-trabajo. La puesta en movimiento de fases del proceso productivo anteriormente integradas en una misma razón social y hasta en un mismo establecimiento, la tendencia a la reducción y dispersión del plantel de trabajadores/as, y la predisposición generalizada al desplazamiento con vistas a mejorar las condiciones de vida, nos han aportado tres ejes desde los que abordar el proceso de deslocalización en la industria de la confección de indumentaria argentina.

A partir del recorrido propuesto constatamos, en línea con Cortés (1988), que la reestructuración del sector ha trasladado la producción de la fábrica al domicilio habilitando, producto de este proceso, el trabajo clandestino y la intensificación de la economía no registrada. 
Por otro lado, la expansión del trabajo en condiciones similares a la servidumbre presiona a la baja a aquellas propias del ámbito registrado. Del mismo modo que la desocupación, en términos de ejército de reserva, incide en el conjunto de asalariados/as ocupados/as, la expansión de condiciones precarias de trabajo en el ámbito no registrado disciplina y retrae los límites de exigencia pensables dentro del campo formal. La escasa articulación entre trabajadores/as del sector -registrados y no registrados- y la exigua intervención estatal y sindical abonan el terreno sobre el que se erige el crecimiento de la plusvalía absoluta.

Como se ha detallado en estas páginas, la desintegración del proceso productivo ha incrementado la precarización del trabajo -revitalizando el trabajo a domicilio y el empleo clandestino- con el objetivo de adaptarse a ritmos de producción más veloces y flexibles. La migración indocumentada y la atomización de las unidades productivas son factores que se conjugaron en la conformación de un colectivo de trabajadores sumamente vulnerable, desarticulado e inerme para refrenar el despliegue avasallante del capital.

\section{Referencias}

Abramo, L., Rodríguez, E. y Rossignotti, G. (2004). Cadenas productivas, trabajo a domicilio y organización sindical. Lima, Perú: Organización Internacional del Tabajo OIT.

Adúriz, I. (2009). La industria textil en Argentina. Su evolución y sus condiciones de trabajo. FOCO - Foro Ciudadano de Participación por la Justicia y los Derechos Humanos del Instituto para la Participación y el Desarrollo (INPADE). Buenos Aires, Argentina: Instituto para la Participación y el Desarrollo. Recuperado en http://foco.org.ar/documentos/Documentos\%20de\%20trabajo/La\%20industria\%20textil\%20en\%20Argentina.pdf

Altamira, C. (2001). La naturaleza del trabajo en el fin de siglo. Diálogos: Educación y formación de personas adultas, 28, 105-105.

Arndt, S. W. (1997). Globalization and the Open Economy. North American Journal of American Economics and Finance, 8 (1), 71-79. 
Baldone, S., Sdogati, F. y Tajoli, L. (2001). Patterns and determinants of international fragmentation of production: Evidence from outward processing trade between the EU and Central Eastern European Countries. Weltwirtschaftliches Archiv, 137 (1), 80-104.

Belini, C. (2008). Una época de cambios: la industria textil argentina entre dos crisis, 19141933. Estudos Ibero-Americanos, 34, 31-48.

Benencia, R. (2009). El infierno del trabajo esclavo. La contracara de las 'exitosas' economías étnicas. Avá. Revista de Antropología, 15, 43-72. Recuperado en http://www.scielo.org. ar/scielo.php?pid=S1851-16942009000200002\&script=sci_arttext

Caggiano, S. (2005). Lo que no entra en el crisol. Inmigración boliviana, comunicación intercultural y procesos identitarios. Buenos Aires, Argentina: Prometeo Libros.

Cámara de Indumentaria de Bebés y Niños (CAIBYN) (2010). El sector de la indumentaria infantil en la Argentina. Informe de trabajo correspondiente al mes de febrero. Buenos Aires, Argentina: CAIBYN.

Cámara Industrial Argentina de la Indumentaria (CIAI) (2014). Evolución del sector de indumentaria durante el año 2013 y perspectivas para el 2014. Resultados del relevamiento realizado durante el mes de enero de 2014. Informe de trabajo correspondiente al mes de febrero. Buenos Aires, Argentina: CIAI.

Cerrutti, M. (2009). Diagnóstico de las poblaciones de inmigrantes en la Argentina. Serie de Documentos de la Dirección Nacional de Población. Buenos Aires, Argentina: Dirección Nacional de Población, Secretaría del Interior, Ministerio del Interior.

China Labour Bulletin (27 de junio de 2013). Migrant workers and their children. Recuperado de http://www.china-labour.org.hk/en/node/100259

China.org (26 de febrero de 2010). Argentina inicia investigaciones antidumping sobre ropa china importada. Recuperado de http://spanish.china.org.cn/economic/txt/201002/26/content_19483241.htm

Cortés, R. (1988). El trabajo clandestino en la industria del vestido. CIAT-OIT-Ministerio de Trabajo y Seguridad Social (Ed). El empleo precario en Argentina, Vol. 2. Lima, Perú: CIAT-OIT. 
De Génova, N. (2002). Migrant 'Illegality' and Deportability in Everyday Life. Annual Review of Anthropology, 31, 419-447.

D’Ovidio, M. (2007). Quién es quién en la cadena de valor del sector de indumentaria textil. Informe realizado por la Fundación El Otro (www.elotro.org.ar) en alianza con interrupción (www.interrupcion.net).

Engels, F. (1881 [2013]). El sistema de trabajo asalariado. The Labour Standard. Marxists Internet Archive (www.marxists.org).

Feenstra, R. (1998). Integration of trade and disintegration of production. Journal of Economic Perspectives, 12 (4), 31-50.

Germani, G. (2010). La inmigración masiva y su papel en la modernización del país. En C. Mera, y J. Rebón, J (Coord.), Gino Germani. La sociedad en cuestión. Antología comentada (pp. 440-543). Buenos Aires, Argentina: Consejo Latinoamericano de Ciencias Sociales. CLACSO.

Gallart, M. A. (2006). Análisis de las estrategias de acumulación y de supervivencia de los trabajadores ocupados en la rama de textiles y confecciones. En Organización Internacional del Trabajo OIT, Informalidad, Pobreza y Salario Mínimo, Programa Nacional de Trabajo Decente Argentina 2004-2007, (pp. 127-182). Buenos Aires, Argentina: OIT.

Goldberg, A. (25-28 de octubre de 2010). Precariedad laboral y explotación ilegal de trabajadores inmigrantes bolivianos en talleres textiles clandestinos del Área Metropolitana de Buenos Aires: su impacto en la salud. Ponencia presentada en I Seminário Internacional de História do Trabalho Universidade Federal de Santa Catarina, Florianópolis, Brasil.

Harvey, D. (2007). Espacios del Capital. Hacia una Geografía Crítica. Madrid, España: Akal.

Hijzen, A., Görg, H. y Hine, R. (2003). International fragmentation and relative wages in the UK. Institute for the Study of Labor (IZA) Discussion Papers, 717.

Hummels, D.; Ishii, J. y Yi, K. (2001). The Nature and Growth of Vertical Specialisation in World Trade. Journal of International Economics, 54, 75-96.

Instituto Nacional de Educación Tecnológica (INET) (2008). El Sector de la Indumentaria en Argentina. Serie de informes sectoriales. Recuperado en www.inet.edu.ar 
Irigoin, A. (1984). La evolución industrial en Argentina (1870 - 1940). Revista Libertas del Instituto universitario ESEADE, 1. Recuperado en http://www.eseade.edu.ar/servicios/ Libertas/49_6_Irigoin.pdf

Jelin, E., Mercado, M. y Wyczykier, G. (1998). El trabajo a domicilio en la Argentina. Informe final de investigación. Santiago, Chile: OIT.

Jones, R. W. y Kierzkowski, H. (1990). The Role of Services in Production and International Trade: A Theoretical Framework. En R.W. Jones y A. Krueger (eds.), The Political Economy of International Trade (pp. 31-48). Oxford: Basil Blackwell.

Kosacoff, B. (Ed.). (2004). Evaluación de un escenario posible y deseable de reestructuración y fortalecimiento del Complejo Textil argentino. Buenos Aires, Argentina: CEPAL.

Leamer, E. (1998). In Search of Stolper-Samuelson Linkages between International Trade and Lower Wages. En S.M. Collins (ed.), Imports, Exports, and the American Worker (pp. 141-203). Washington D.C, Estados Unidos: Brookings Institution Press.

Lebowitz, M. (2005). Más allá del Capital. La economía política de la clase obrera en Marx. España: Akal.

Lieutier, A. (2010). Esclavos: los trabajadores costureros de la ciudad de Buenos Aires. Buenos Aires, Argentina: Retórica Ediciones.

Marshall, A. (1990). Formas precarias de trabajo asalariado: Dos estudios en el Área Metropolitana de Buenos Aires. Documento de discusión para el Programa Mercado de Trabajo. Ginebra, Suiza: IILS.

Observatorio de Empleo y Dinámica Empresarial del Ministerio de Trabajo, Empleo y Seguridad Social (OEDE - MTySS). Recuperado el 11 de mayo de 2014 de http://www. trabajo.gov.ar/left/estadisticas/oede/estadisticas_nacionales.asp

Organización Internacional del Trabajo OIT (2000). (28 de octubre de 1996). La mundialización cambia la fisonomía de las industrias de los textiles, el vestido y el calzado. Comunicado de prensa. Recuperado en http://www.ilo.org/global/about-the-ilo/newsroom/ news/WCMS_008432/lang--es/index.htm 
OIT - CEA (2011). Trama productiva urbana y trabajo decente. Estrategias para la cadena productiva textil de indumentaria en áreas metropolitanas. Buenos Aires, Argentina: Oficina de País de la OIT para la Argentina.

Pascucci, S. (2010). Migraciones y clase social. Un análisis crítico de la bibliografía sobre inmigrantes bolivianos en Argentina. Miradas en Movimiento, 4.

Salgado, P. D. (25-28 de octubre de 2010) Inmigración, precariedad laboral y organización sindical: aproximaciones a partir del caso argentino. Ponencia presentada en I Seminário Internacional de História do Trabalho Universidade Federal de Santa Catarina, Florianópolis, Brasil.

-_- (3-5 de agosto de 2011). El trabajo en la industria de la confección: aproximaciones a partir del desenvolvimiento del sector en la última década. Ponencia presentada en $10^{\circ}$ Congreso de la Asociación de especialistas en Estudios del Trabajo (ASET), Buenos Aires, Argentina.

- - - (2012). El trabajo en la industria de la indumentaria: una aproximación a partir del caso argentino. Revista Trabajo y Sociedad, 18, 59-68. Recuperado en http://www.unse. edu.ar/trabajoysociedad/18\%20SALGADO\%20Industria\%20indumentaria\%20.pdf

Sassen, S. (2007). Una sociología de la globalización. Buenos Aires: Katz.

Silver, B. (2005). Fuerzas de trabajo: Los movimientos obreros y la globalización desde 1870. España: Akal.

The Economist (29 de abril de 2014). La gran y atropellada transición de China. Recuperado en http://www.infomovilnews.com/?p=34206

Tomei, M. (1999). El trabajo a domicilio en países seleccionados de América Latina: una visión comparativa. Cuestiones de desarrollo. Documento de discusión 29, OIT. 